Rhode Island College

Digital Commons @ RIC

$12-1-2019$

\title{
Increasing Participation in the American Nurses Association's Healthy Nurse Healthy Nation Grand Challenge in a College Setting
}

Emily Haynes

Follow this and additional works at: https://digitalcommons.ric.edu/etd

Part of the Nursing Commons

\section{Recommended Citation}

Haynes, Emily, "Increasing Participation in the American Nurses Association's Healthy Nurse Healthy Nation Grand Challenge in a College Setting" (2019). Master's Theses, Dissertations, Graduate Research and Major Papers Overview. 315.

https://digitalcommons.ric.edu/etd/315

This Major Paper is brought to you for free and open access by the Master's Theses, Dissertations, Graduate Research and Major Papers at Digital Commons @ RIC. It has been accepted for inclusion in Master's Theses, Dissertations, Graduate Research and Major Papers Overview by an authorized administrator of Digital Commons @ RIC. For more information, please contact digitalcommons@ric.edu. 

INCREASING PARTICIPATION IN THE AMERICAN NURSES ASSOCIATION'S

\section{HEALTHY NURSE HEALTHY NATION GRAND CHALLENGE}

IN A COLLEGE SETTING

A Major Paper Presented

by

Emily Haynes

Approved:

Committee Chairperson

Committee Members

(Date)

(Date)

(Date)

Director of Master's Program

(Date)

Dean, School of Nursing

(Date) 
INCREASING PARTICIPATION IN THE AMERICAN NURSES ASSOCIATION'S HEALTHY NURSE HEALTHY NATION GRAND CHALLENGE

IN A COLLEGE SETTING

\author{
by \\ Emily Haynes \\ A Major Paper Submitted in Partial Fulfillment \\ of the Requirements for the Degree of \\ Master of Science in Nursing \\ in \\ The School of Nursing \\ Rhode Island College
}

2020 



\begin{abstract}
The health status of Americans ranks poorly compared to other nations. The health behaviors of American nurses are worse than the general population. Healthy Nurse Health Nation (HNHN) is the American Nurses' Association's (ANA's) program to encourage healthy lifestyle practices among nurses with the ultimate goal of improving population health by creating a healthier nursing workforce able to be more credible as health coaches. This paper reviews literature on workplace and college health programs, health behaviors of nurses and student nurses, and the idea of nurses as role models. The Logic Model was used to guide implementation of the program and the Ecological Model was used for evaluating the project. This project promoted HNHN in a college setting through in person presentations. Thirty-two students and faculty completed retrospective pre/post surveys, and results showed small self-reported changes in participation. There was an increase from five to 10 members of the RIC HNHN online group. Limitations included limited contact between the researchers and participants, reliance on university email for communication, a lack of graduate student data, and a lack of changes to the environment. The author recommends increasing contact between the researchers and students/faculty and making changes to the environment to make healthy choices the default option. The role of the advanced practice nurse is discussed and includes implications for practice related to education, practice, policy, and research.
\end{abstract}

Keywords: nurses, nursing students, health, wellness, Healthy Nurse Healthy Nation, workplace health, college health 


\section{Acknowledgements}

I would like to thank the people who advised me and supported this project: Lynn Blanchette, Joanne Costello, Maureen Bouris, Wendy Doremus, and Debra Servello. I would also like to thank the students and faculty who participated in this project. 


\section{Table of Contents}

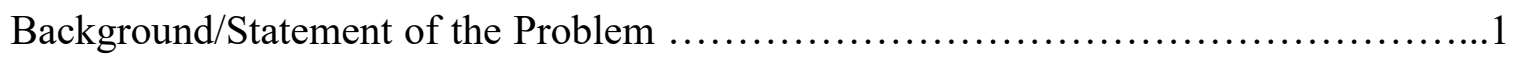

Literature Review...........................................................

Theoretical Framework........................................................ 21

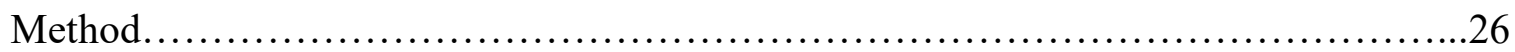

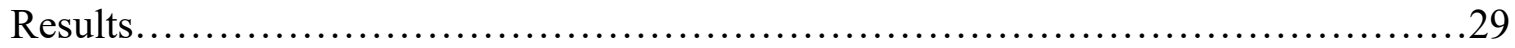

Summary and Conclusions............................................... 31

Recommendations and Implications for Advanced Nursing Practice...................34

References.............................................................. 38

Appendicies.............................................................43 
Increasing Participation in the American Nurses Association's Healthy Nurse Healthy Nation Grand Challenge in a College Setting

\section{Background/Statement of the Problem}

The constitution of the World Health Organization defines health as "a state of complete physical, mental and social well-being and not merely the absence of disease or infirmity" (World Health Organization, 2014, p. 1). American nurses are in a position to help the general population meet this definition of health, but compare poorly in measures of their own health. According to the American Nurses Association (ANA), American nurses are more likely than the general population to be overweight, get inadequate amounts of sleep, and have high levels of stress (ANA, 2018).

Multiple studies have documented poor health of the American population. A 2013 report by the National Research Council and Institute of Medicine explored the health of the U.S. population compared to 16 other high income countries. One of the key findings is a shorter life expectancy of Americans compared to almost all of the other high-income countries. The U.S. also ranks among the highest for prevalence of obesity, diabetes, heart disease, chronic lung disease, and disability (The National Academies, National Research Council and Institute of Medicine, 2013).

The CDC's National Health Statistics Reports provide some useful data on the health behaviors of Americans. A 2011 report used National Health and Nutrition Examination Survey data to estimate how well Americans are following the Dietary Guidelines for Americans 2005. They found that the only categories where Americans were meeting the recommendations were for intake of total grains and intake of meats and beans (U.S. Department of Health and Human Services, Centers for Disease Control 
and Prevention, National Center for Health Statistics, 2011). A 2018 report also used National Health and Nutrition Examination Survey data and reported that only $22.9 \%$ of adults aged 18-64 meet the guidelines for physical activity and muscle-strengthening activities from the 2008 U.S. Department of Health and Human Services federal physical activity guidelines. These guidelines recommend 150 minutes of moderate physical activity per week or 75 minutes of vigorous physical activity per week in addition to muscle-strengthening activities at least two days per week (U.S. Department of Health and Human Services, Centers for Disease Control and Prevention, National Center for Health Statistics, 2018).

Healthy People 2020 has identified a need to improve overall health and wellness with one of the overarching goals being: "Promote quality of life, healthy development, and healthy behaviors across all life stages" (U.S. Department of Health and Human Services, 2018, p. 1). Additional objectives related to health-related quality and life and wellbeing align with the World Health Organization definition of health (2014). According to Healthy People 2020 baseline data, in $201078.8 \%$ of adults reported good or better physical health and $79.1 \%$ of adults reported good or better mental health. Healthy People 2020 identifies a need for improvement in this area and has goals of one percentage point improvement for each of these objectives (U.S. Department of Health and Human Services, 2018).

\section{Identification of Clinical Problem}

Nurses have a unique opportunity to influence the health of the general population by educating patients about health promotion and by modeling healthy lifestyle behaviors. According to the ANA's Code of Ethics for Nurses, nurses have a professional 
duty to promote their own health, safety, and well-being. It is essential for nurses to model the health behaviors that they are teaching including eating a healthy diet, exercising, getting adequate rest and maintaining balance in life through family relationships, leisure time activities, and addressing spiritual needs (ANA, 2015).

Between October 2013 and October 2016, the ANA conducted an online survey of nurses called the Health Risk Appraisal in order to collect data on health, wellness, and safety of nurses and to help respondents identify areas where they could make healthy lifestyle changes. According to data from the ANA Health Risk Appraisal, the average BMI of nurses who responded to the survey was 27.6 which places them in the overweight range. Only $16 \%$ of respondents reported eating at least five servings of fruits and vegetables per day (ANA, 2018).

The ANA advocates for the health and safety of nurses both professionally and personally and encourages nurses to make healthy lifestyle choices through their Healthy Nurse Healthy Nation (HNHN) Grand Challenges. The Grand Challenges are online 10day challenges that aim to engage nurses to improve their health in the key areas of physical activity, rest, nutrition, quality of life, and safety. Participants are asked to complete the Health Risk Appraisal survey and then choose areas to focus on to make health commitments and participate in challenges. The Grand Challenge encourages participants to post plans for healthy lifestyle changes and participate in discussion boards. Past challenges have included, for example: incorporate whole grains, cut down on fast food, step it up, curb your sweet tooth, hydration, and healthy sleep. The goal is that healthier nurses will be better role models for healthy behavior and be able to better educate patients and the public about healthy lifestyles (ANA, 2018). 
Organizations including workplaces, schools of nursing, state nurses' associations, and professional nursing organizations are encouraged to join $\mathrm{HNHN}$ as organizational partners. Some of the schools of nursing that have been successful in implementing HNHN are featured on blog posts. In addition to encouraging students and faculty to participate in HNHN, schools of nursing have implemented wellness committees, yoga classes, step challenges, stress relief walks, and back safety and exercise programs (ANA, 2018). Rhode Island College School of Nursing was already an organizational partner, but prior to this project there were only five members in the online group. This goal of project was to increase participation in HNHN at Rhode Island College School of Nursing.

\section{Purpose Statement and Project Type}

The purpose of this project was to increase participation in the ANA's HNHN Grand Challenge in a college setting with students and faculty. It addressed the question: Does promoting participation in HNHN through in-person presentations for Rhode Island College School of Nursing undergraduate students, RN to BSN students, graduate students, and faculty increase the number of participants who join the RIC HNHN online group and participate in a minimum of one HNHN Grand Challenge compared to preintervention? This program development project expanded on the already established HNHN Grand Challenge in a college setting. 


\section{Literature Review}

A literature review was conducted using CINAHL Plus with Full Text, Academic Search Complete, and JSTOR. Key words searched included: nurses, nursing students, health, obesity, workplace, physical activity intervention, nutrition intervention, role models, college students, college health, self-care, curriculum, and Healthy Nurse Healthy Nation. Selections were published between 2011 and 2018 and were limited to peer reviewed articles published in English.

\section{Workplace Health and Wellness Interventions}

Goetzel et al. (2014) provided expert opinion for the American College of Occupational and Environmental Medicine in a compilation of evidence on the effectiveness of workplace health promotion programs to answer the question: do workplace health promotion programs work? The paper identified the elements necessary for workplace health promotion programs to be successful. These elements included: the program must have clear goals which may be related to engaging employees, making employees more aware of their own health, reducing behavioral risk factors for disease, reducing the company prevalence of chronic disease, reducing injuries and absences, or providing a return on investment for the employer. The article stated that health promotion programs should use an appropriate framework to evaluate program success. They should include an evaluation of the program structure, delivery process, and the expected outcomes. The authors stated that programs that are not comprehensive and may include only a health risk assessment or health promotion web site are usually not effective. The authors recommend Healthy People 2010's elements for comprehensive workplace health promotion which include: 
(1) Health education, focused on skill development and lifestyle behavior change along with information dissemination and awareness building. (2) Supportive social and physical environments, reflecting the organization's expectations regarding healthy behaviors and implementing policies promoting healthy behaviors. (3) Integration of the worksite program into the organization's benefits, human resources, infrastructure, and environmental health and safety initiatives. (4) Links between health promotion and related programs like employee assistance. (5) Screenings followed by counseling and education on how to best use medical services for necessary follow-up (Goetzel, et al., 2014, p. 929-930). They discussed the importance of creating a culture of health describing it as an environment where healthy choices are the easy choices. This can be done by making healthy foods more accessible in the cafeteria and vending machines or by serving healthy foods at meetings. Creating a culture of health also means that leaders of an organization care about the health of employees. The authors answered the original question that workplace health promotion programs can be successful if they are implemented appropriately with evidence based interventions (Goetzel et al., 2014).

Malik, Blake, and Suggs (2014) conducted a systematic review to evaluate results of workplace health promotion interventions. A total of 58 individual studies were included in the systematic review. Studies were grouped into three categories of interventions that included (1) physical activity/exercise interventions, (2) counselling/support interventions, and (3) health promotion messages/information interventions. There was no statistical analysis due to heterogeneous study designs. Of the six physical activity interventions, two studies had statistically significant results 
related to increasing physical activity levels using measures of daily step counts, weekly physical activity and others. Thirteen studies were considered counseling/support interventions using different combinations of telephone, face to face, individual, or group counseling, and eight of those had statistically significant increases in physical activity. Thirty-nine of the studies provided health promotion messages or information, and 22 of these had statistically significant results. Some of the studies used the Transtheoretical Model to tailor interventions to a specific stage of change and these interventions were generally found not to be any more effective than interventions that were not tailored. The authors conclude that there was insufficient evidence to determine whether workplace health promotion interventions are effective for increasing physical activity. Many of the individual studies had methodological limitations including relying on selfreported activity levels and using non-validated questionnaires. Many of the studies also used multicomponent interventions so there was no way to distinguish which intervention increased physical activity. The researchers recommend more research on workplace health promotion interventions (Malik et al., 2014).

White et al. (2016) published a synthesis of systematic reviews on the effects of workplace physical activity interventions on reducing disability which is relevant to HNHN's safety domain as HNHN aims to reduce workplace injuries for nurses (ANA, 2018). There was no meta-analysis because of different study designs and outcomes measured. Eighteen systematic reviews were included and were grouped into categories of intervention goals including: (1) reducing work absence, (2) improving work productivity, or (3) reducing the financial impact. The paper separated interventions for workers who were at work at baseline and workers who were out of work due to injury. 
For workers who were at work at baseline, evidence showed that simple physical activity interventions (interventions with one or two foci) may reduce work absence, but results were mixed for improving work productivity and financial outcomes. Complex physical activity interventions, for workers who were at work at baseline, found mixed or inconclusive results for all three of the outcome measures. For the workers who were out of work at baseline, simple physical activity interventions did improve sick leave outcomes for workers with back pain. Four of the five studies reviewing complex interventions for workers who were out of work found improved results for sick leave outcomes. None of the studies measured work productivity outcomes or financial outcomes for workers who were out of work. Limitations included probable volunteer bias and publication bias. The authors reported that more high-quality research is needed on this topic (White et al., 2016).

Kent, Goetzel, Roemer, Prasad, and Freundlich (2016) conducted a review of literature, focus groups with subject matter experts, and workplace site visits to help establish best practices for promoting healthy workplaces. A total of 18 subject matter experts from academia, health promotion companies, research organizations, consulting firms, and nonprofits participated in focus groups addressing organizational structure, implementation, and measurement and evaluation of workplace wellness programs. The authors chose a convenience sample of nine employers that all offered good programs and were able to demonstrate results. Site visits lasted 6-7 hours and included interviews with senior managers, focus groups with team members involved in coordinating the wellness programs, and focus groups with line workers including participants and 
nonparticipants in wellness programs. Site visits also included worksite tours (Kent et al, 2016).

According to Kent et al. (2016), the most successful workplace health promotion programs focused on building a culture of health and strategic communications. Organizations with successful health promotion programs need to do more than just isolated acts of wellness. They need to create a culture of health by placing value on employee health and wellbeing. Successful organizations altered the physical environment to promote health by making healthy foods more available and providing onsite recreation facilities and clinics. Creating a culture of health also requires altering the social environment to promote health. To create a positive social environment, organizations need support and participation from senior leadership and middle managers and peer support through team challenges or family member participation. The final element necessary for creating a culture of health involves employee involvement in planning and implementing wellness programs. In addition to creating a culture of health, successful organizations used strategic communications to promote workplace wellness. Communication should be modeled on a behavior change theory and should clearly convey the goals of the wellness program. Communication must be tailored and targeted to employees of different age, sex, or education level. Successful communication should be multi-channeled utilizing email, newsletters, break room posters, and word of mouth. Communication must also be bidirectional with leadership seeking feedback from employees. The authors conclude by discussing how a culture of health creates more productive employees and more financially successful organizations (Kent et al., 2016). 


\section{Nurses as a Vulnerable Population Related to Health Risks}

A systematic review by Fronteira and Ferrinho (2011) reviewed experimental and observational studies on the physical health of nurses. A total of 187 individual studies published between 1984 and 2008 were included in this review. There was no metaanalysis due to heterogeneous study designs so results were presented in narrative form. Musculoskeletal disorders were discussed first and evidence showed that nurses were more affected than individuals in other occupations except for professional drivers, maintenance workers, and nursing assistants. Studies on blood borne pathogens had mixed results with some showing that nurses were more exposed than other healthcare workers and others showing that nurses were less exposed. Studies on cancer in nurses mostly used data from national cancer registries and found a higher incidence of breast cancer among female nurses compared to other healthcare workers but not compared to the general population. The authors suggested that this may be due to occupational exposures to carcinogens or due to increased breast cancer screening among nurses. There was no increased risk identified for any of the other cancers studied. There was not enough evidence to determine if nurses have a greater risk for hypertension or cardiovascular disease. Many of the studies on tuberculosis found that nurses working in high risk settings including hospitals, pulmonary units, TB units, and HIV units had a higher risk of TB. Studies showed a higher prevalence of occupational allergic dermatitis in nurses. Most of the research on sleep disorders did not compare nurses to other occupations, but one study that did compare nurses and nursing assistants found that nursing assistants had more sleep disorders than nurses. One study on diabetes found that nurses were not at greater risk for diabetes than other healthcare workers. One limitation 
identified is that the systematic review was limited to physical health and excluded mental health and quality of life (Fronteira \& Ferrinho, 2011).

An integrative review by Nicholls, Perry, Duffield, Gallagher, and Pierce (2016) explored workplace barriers and facilitators to healthy eating for nurses. A total of 26 quantitative and qualitative studies were included in the review. Quality appraisals were not high, indicating that individual studies had methodological limitations, but all of these studies were included because of their relevance and the authors considered the quality when drawing conclusions. Long working hours were found to be a barrier to healthy eating because it does not allow time for preparing healthy meals and leaves nurses with little energy for meal preparation. Shift work was a barrier because it interfered with normal eating with some studies finding that shift workers tended to eat more and snack more at work rather than eating full meals. Workplace stress was not clearly a barrier or facilitator with some studies showing that workplace stress led to emotional eating as a coping mechanism, but a different study found that nurses who reported more stress reported healthier diets. Environmental factors in the workplace made healthy eating difficult on evening and night shifts when healthy food options were limited or not available. The authors also identified that the environment could also make it difficult for nurses who bring their own food because refrigerators and microwaves may be unavailable or too far from the work area. Some work sites did facilitate healthy eating by making cafeterias look attractive and making healthy foods more available. This study identified that sharing meals with colleagues could be either a barrier or facilitator because conversations about diet could motivate healthy eating, but social gatherings often involved unhealthy foods. Personal knowledge and self-efficacy also influenced 
eating habits. Studies found that overweight nurses often did not perceive themselves to be overweight or obese. One study found that nurses did not score high on scales measuring knowledge of obesity prevention. The authors discussed the potential for healthcare facilities to alter their environments to improve the health of nurses (Nicholls et al., 2016).

A qualitative study by Power, Kiezebrink, Allan, and Campbell (2017) used the Theoretical Domains Framework to explore barriers and enablers to healthy eating and physical activity for nurses. The Theoretical Domains Framework is a synthesis of behavior change theories described by Michie, Johnston, Abraham, Lawton, and Parker (2005) and given the name by Cane, O'Connor, and Michie (2012). This study by Power et al., (2017) had 16 nurses from one hospital in Scotland participate in semi-structured interviews. Themes were grouped into broad categories of environmnetal factors, interpersonal factors, and intrapersonal factors. The workplace environment was found to be more of a barrier to healthy eating with unhealthy foods easily available and healthy foods described as being limited. Shift work and long working hours were reported as barriers to healthy eating, but some participants reported that shift work enabled them to participate in physical activity. Interpersonal factors included social influences from family, friends, and colleagues and were reported to be both positive and negative. Intrapersonal factors were both barriers and enablers to healthy eating and physical activity. Perceived intrapersonal enablers included beliefs about consequences of actions, emotions, goals, reinforcement or expectations of rewards, knowledge, and optimism. Perceived intrapersonal barriers included distractions from holidays, special occasions or busy work shifts, and beliefs and capabilities. The authors suggested that this data can be 
useful in planning behaviour change techniques in interventions for nurses. A limitation identified by the authors was that nurses' perceptions of their behaviors may not represent their actual eating and physical activity behaviors (Power et al., 2017).

\section{Nurses as Role Models for Healthy Behavior}

There are varying opinions in literature about whether nurses should be expected to be role models for healthy lifestyles. The argument supporting this idea is that nurses must practice healthy behaviors in order to effectively encourage behavior change in patients and that patients may imitate healthy behaviors of nurses that they respect. The argument against this idea is that patients may have difficulty relating to nurses who they perceive as having healthier lifestyles than their own (Kelly, Wills, Jester, and Speller, 2016). Kelly et al., (2016) used a modified Policy Delphi study with stakeholders in nursing education, nursing policy, workforce development, service users, student nurses, and practicing nurses. The goal was to better understand expectations about whether nurses should be expected to model healthy behaviors. There were a total of 25 participants. The first round of data collection consisted of telephone interviews with 11 open ended questions. Data from these interviews were used to develop themes and attitude statements for use in round two of data collection. Round two used an online questionnaire. The five key themes evaluated in round two were: (1) to be more credible in health promotion, (2) to take personal responsibility for their health, (3) because personal health has an impact on the quality of patient care, (4) as a professional duty, or (5) to counteract unhealthy social norms in the workforce. The idea that nurses should be expected to model healthy behavior was somewhat supported by all groups except practicing nurses and student nurses who believed that role modeling healthy behaviors 
was not necessary. There was high consensus among participants in all groups of stakeholders on the statement related to visibly unhealthy nurses being perceived as less credible when giving health advice. There was higher consensus among participants in all groups of stakeholders on the statement that employers should take responsibility for nurses' health compared to the statement that nurses should take more responsibility for their own health. Service users were more likely to agree that visibly unhealthy nurses may be perceived by patients as less competent nurses. There was low agreement that nurses should be expected to meet physical health requirements beyond being able to perform their jobs. The authors discussed the need for employers to take more responsibility for the health of nurses rather than just victim blaming. The study was limited by participant dropout between the first and second rounds of data collection, but dropout was distributed across all stakeholder groups (Kelly et al., 2016).

Kelly, Wills, and Sykes (2017) conducted an integrative review to evaluate evidence supporting the belief that nurses should model healthy behaviors. The review answered the questions: "Do nurses' personal health behaviors impact on their health promotion practices?" and "Do nurses' personal health behaviors impact on patient responses to a health promotion message?" The review used the Logic Model as the theoretical framework to address these questions. Twenty-nine studies with heterogeneous designs met inclusion criteria and addressed the first question related to nurses' health behaviors and health promotion practices. Two studies addressed the second question related to nurses' health behavior and patient responses to health promotion education. Twenty-two studies addressed smoking and health education and found mixed results with some studies reporting that non-smokers addressed smoking 
cessation more frequently and others reported no difference in frequency of addressing smoking cessation between nurses who smoked and nurses who didn't smoke. One study related to diet found that nurses' own diets did affect their confidence in their healthy eating advice. Studies related to weight found mixed results on whether nurses' own weight impacted their self-efficacy in addressing weight with patients. Studies related to physical activity had mixed results with some reporting that more physically active nurses recommended physical activity more frequently and others reporting no difference in physical activity recommendations from physically active and physically inactive nurses. Several studies reported nurses' perceived self-efficacy to be the best predictor of consistent smoking cessation counseling with some studies finding that nonsmokers reported greater self-efficacy. Studies suggested that nurses' confidence in health promotion was influenced more by their training than by personal behavior. There were conflicting opinions on the credibility of nurses who engage in unhealthy behaviors. The authors conclude that there is not enough evidence to argue that nurses who engage in healthy behaviors more frequently engage in health promotion or are more credible as health coaches. More research is needed on patient responses to health promotion education from nurses (Kelly et al., 2017).

The concept of nurses as role models in health promotion was explored and clarified in a concept analysis study by Darch, Baillie, and Gillison (2017). They integrated concepts from a literature review and qualitative data from focus groups and an individual interview to clarify the concept of role modeling. According to this concept, the defining attributes of role modeling healthy behavior included being an exemplar, portraying a healthy image, and championing health and wellness. Personal attributes that 
role models should possess include "being caring, non- judgmental, trustworthy, inspiring and motivating, self-caring, knowledgeable and self-confident, innovative, professional and having a deep sense of self" (Darch et al., 2017, p. 986). The authors were unable to conclude whether an imperfect role model who may have more behaviors in common with their patients, can be effective in promoting health (Darch et al., 2017).

\section{College Student Health and Wellness Interventions}

A systematic review by Maselli, Ward, Gobbi, and Carraro (2018) reviewed randomized controlled trials and non-randomized controlled trials on physical activity interventions among university students. Although 27 studies met inclusion criteria and 16 of those studies reported increases in physical activity levels, the risk of bias in individual studies was high so conclusions were limited. Results were presented in narrative form only. The majority of the effective studies used multicomponent interventions. However, many of the same components of the effective interventions were also used in the ineffective interventions so it is unclear which components of the interventions were most effective. These studies all focused on interventions at individual and interpersonal levels so it is unclear how environmental influences may have interfered with results. Some studies used only an online intervention and were generally less effective than studies with a face to face component. Few of the studies included participating in physical activity as part of the intervention and the authors believed that would be useful. The authors determined that better quality studies with follow up longer than 12 months were needed (Maselli et al., 2018). 


\section{Health Behaviors of Student Nurses}

Blake, Malik, Mo, and Pisano (2011) conducted a cross-sectional survey of nursing students at a National Health Service hospital in England to assess their levels of physical activity, smoking, alcohol behavior, dietary practices, and general health. Only $45.98 \%$ of the 325 participants met the recommendation for 30 minutes of physical activity five times per week. These participants were considered active and participants not meeting this recommendation were considered inactive. Barriers to physical activity were assessed and the most common barriers were lack of time, cost of participating, and feeling tired. Other questions were stratified by activity level and active participants responded more positively to most other questions. The average BMI was 24 . Only $39.36 \%$ of participants reported not getting seven hours of sleep per day the majority of the time. The prevalence of smoking in this sample was $18.8 \%$, and was more common among the inactive participants compared to active participants. The majority $(58.5 \%)$ of participants perceived their diets to be healthy. However, $76.8 \%$ of participants failed to consume five servings of fruits and vegetables per day and 53.9\% of participants reported consuming foods high in fat and sugar. Active participants were more likely to meet the recommendation for fruits and vegetables. About half of the participants were aware of the recommended maximum daily alcoholic beverages, but two thirds reported more than four alcoholic drinks in one day when drinking. The authors discuss the need to increase access to healthy foods in the workplace and create opportunities for physical activity in the workplace. According to the authors binge drinking is a problem in the general population and nurses could be role models if they reduced their risky drinking. The study was limited by nonresponse bias and by relying on self-report data. The authors 
concluded that student nurses had relatively poor health promotion behaviors and should have education on the importance of maintaining their own health (Blake et al., 2011).

A cross-sectional survey by Blake and Harrison (2013) conducted at a university teaching hospital in England assessed health practices of student nurses and their attitudes about role modeling healthy behaviors for patients. Of the sample of 540 student nurses, $24 \%$ were either overweight or obese. Many participants reported not meeting recommendations for healthy behaviors assessed in this survey with $46.9 \%$ not meeting the recommendation for 30 minutes of physical activity five days per week and $72.8 \%$ not consuming at least five servings of fruits and vegetables per day even though $67.2 \%$ of the sample reported that they considered their diets to be healthy. Smoking and alcohol were also assessed and $16.8 \%$ of participants reported being current smokers. Most participants reported some alcohol consumption with $40.4 \%$ reporting drinking $2-4$ times per month and 33.5\% reporting drinking 2-3 times per week. In addition, $40 \%$ of participants reported consuming five or more drinks on each occasion. The second part of this survey assessed student attitudes about role modeling healthy behavior and compared responses to weight category. Student nurses generally agreed that their appearance affected how patients perceived them as nurses, but students who were overweight or obese were less likely to agree with this. Most participants agreed that patients would be more likely to follow advice if the nurse appeared to follow their own advice. Most participants agreed that maintaining a positive image of the nursing profession is important with participants agreeing that nurses should not be allowed to smoke while in uniform and that maintaining a healthy weight for nurses should be important. Most of the participants agreed that patients would be able to better relate to nurses they perceived 
as a "real person" rather than a perfect role model. The majority of participants agreed that nurses should consider themselves role models for healthy behaviors, but overweight and obese students were less likely to agree. The student nurses believed that presenting a professional nursing image as healthy role models is important, but their personal behaviors were not consistent with this attitude. The author discusses the need for health promotion education for student nurses (Blake \& Harrison, 2013).

Unpublished research by Rock (2018) used a quantitative and qualitative survey to assess health promotion practices of undergraduate nursing students at Rhode Island College. The sample included 141 participants. A 52 item Health-Promoting Lifestyle Profile II questionnaire was used and contained questions related to health responsibility, physical activity, nutrition, spiritual growth, interpersonal relations, and stress management. The highest scores were in the category of spiritual growth with an average score of 3.01 on a four point Likert scale meaning that the majority of participants reported that they often or routinely engaged in positive spiritual growth by agreeing to statements related to finding a purpose in life and working on long term goals. The next highest average scores were in the interpersonal relations category with an average score of 2.99 with questions related to effective communication, developing meaningful relationships, and spending time with close friends. The section on health responsibility contained questions related to being educated about health promotion and taking preventative action to prevent disease. The average score for this section was 2.26 showing that the majority of participants were only sometimes taking appropriate responsibility for their health. The physical activity section had an average score of only 2.2 and the nutrition section had average score of 2.48 on the four point Likert scale. The 
stress management section for nursing students had an average score of 2.23 . This survey also included open ended questions to assess barriers to health promotion specific to student nurses and the most common barriers identified were lack of time, stress, and guilt (Rock, 2018).

Based on this literature review, it is evident that more research is needed on workplace wellness interventions and the health of nurses and students nurses (Malik et al., 2014; White et al., 2016). Workplace wellness programs may be effective if they are well planned and have clear goals (Goetzel et al., 2014; Kent et al., 2016). Nurses face environmental risk factors in their work environments that make healthy choices difficult and may expose them to infectious diseases (Fronteira \& Ferrinho, 2011; Nicholls et al., 2016; Power et al., 2017). Some research has found that nurses lack adequate knowledge on health promotion (Nicholls et al., 2016). There is some consensus among nurses, employers, and student nurses that multiple stakeholders should be responsible for improving the health of nurses, but data on effective interventions for nurses is limited (Kelly et al., 2016; Nicholls et al., 2016). Nursing students report poor personal health practices and more data is needed on incorporating health promotion into nursing school curriculums (Blake et al., 2011; Blake \& Harrison, 2013; Maselli et al., 2018; Rock, 2018). Whether improving the health of nurses with improve the health of the general population is still unclear (Darch et al., 2017; Kelly et al., 2017). 


\section{Theoretical Framework}

The Ecological Model was used as the framework for this program development project. The Ecological Model used for health promotion was adapted from Bronfenbrenner's (1977) article Toward an Experimental Ecology of Human Development. Bronfenbrenner suggested that psychologists needed to change the way they studied children. Previous research that studied the interactions of one parents and one child together did not consider how the environment could influence actions of one participant and those actions could influence the actions of the other participant. Bronfenbrenner suggests that human development is influenced by the ecological environment which includes a microsystem, mesosystem, exosystem, and macrosystem. The microsystem is the relationship between the developing person and one setting of their environment such as home or school. The mesosystem is the system of microsystems influencing a developing person. The exosystem in comprised of structures within a society which may include neighborhoods, government agencies, mass media, transportation facilities, or informal social networks. The macrosystem includes the written and unwritten rules that influence culture and govern society (Bronfenbrenner, 1977).

The use of an ecological perspective for public health and health promotion interventions was first documented by McLeroy, Bibeau, Steckler, and Glanz (1988). This model moves away from older health promotion models that focused on individuals only and could be seen as victim-blaming. An Ecological Model considers influences of intrapersonal and interpersonal factors in addition to influences from institutional, 
community, and public policy factors. Interventions using an Ecological Model target multiple levels of influences (McLeroy et al., 1988).

Health promotion interventions at the intrapersonal level focus on changing individual health behavior choices through changes in knowledge, attitudes, or skills. Alternately, interpersonal influences on health may include family, friends, or work groups. Interventions at the interpersonal level seek to modify social influences or social norms that encourage or promote negative behaviors. Organizational influences are important because people spend a large amount of their time in schools or workplaces and influences can be either positive or negative. Applying the Ecological Model, workplace health promotion programs should focus not only on creating healthy employees, but also on creating healthy workplace environments. Communities can be defined by geographic location, patterned social interactions, or political entities. Relationships between community organizations can help or hinder health promotion efforts as they compete for limited resources. According to ecological theory concepts, community agencies such as churches may be appropriate intervention sites for health promotion programs. Communities also control whether health promotion issues become part of the public agenda and may block issues that have potential economic consequences. Public policy is part of the Ecological Model because policies such as restricting smoking in public places or taxes on cigarettes can cause behavior changes. Public policy may also promote or restrict access to health promotion programs (McLeroy et al., 1988).

The American College Health Association (2018) uses the Ecological Model as the framework for their Healthy Campus 2020 Initiative which uses objectives similar to 
the Healthy People 2020 objectives. Healthy Campus 2020 considers the influence of the campus environment on the health of students, faculty, and staff. Objectives are separated into categories for students and faculty/staff and are related to nutrition and weight status, physical activity, stress management, tobacco, health impediments to academic performance, health communication, injury and violence prevention, mental health, sexually transmitted diseases, substance abuse, and immunizations. The diagram below is the American College Health Association's representation of the Ecological Model and helps explain the influences of intrapersonal, interpersonal, institutional, community, and public policy factors on health behaviors (American College Health Association, 2018).

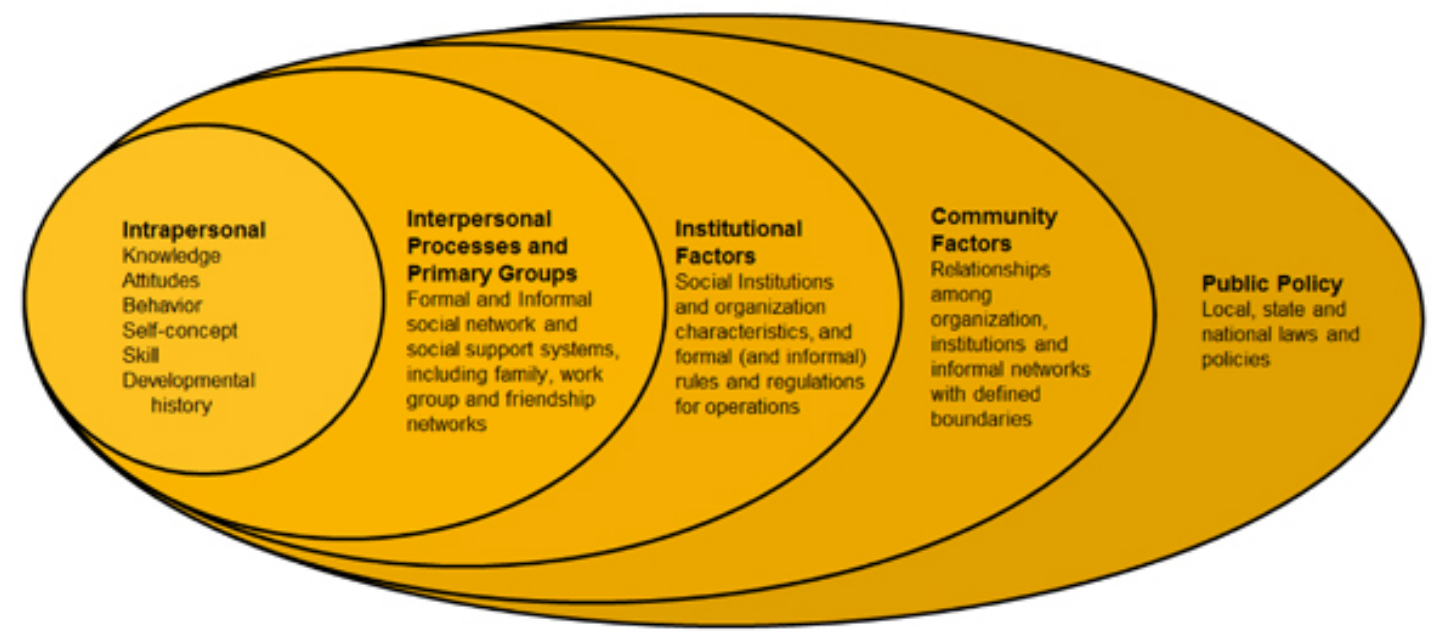

Figure 1. Ecological Model showing influences on health behavior. (American College Health Association, 2018).

This program also used parts of the Logic Model from the W.K. Kellogg Foundation (2004). The Logic Model is a program planning and evaluation tool used to visually represent relationships between resources and inputs, activities, outputs, outcomes, and impacts of a program. It is useful during any phase of program planning, 
implementation, or evaluation and should be adapted for each project to focus more on theory, activities, or outcomes. Inputs may include human, financial, or organizational stakeholders and resources. Activities are the intervention being implemented with these resources. Outputs are the immediate changes resulting from the intervention. Resources, activities, and outputs for the HNHN program at Rhode Island College are identified in the chart below. The Logic Model also includes outcomes and impact, but these will not be measured due to time constraints for the HNHN program. Outcomes are the changes in individual participants' behavior, knowledge, or skills and can be measured as soon as one year after the activities. Impact is the changes in organizations, communities, or systems and occurs within seven to ten years of the program activities (W. K. Kellogg Foundation, 2004).

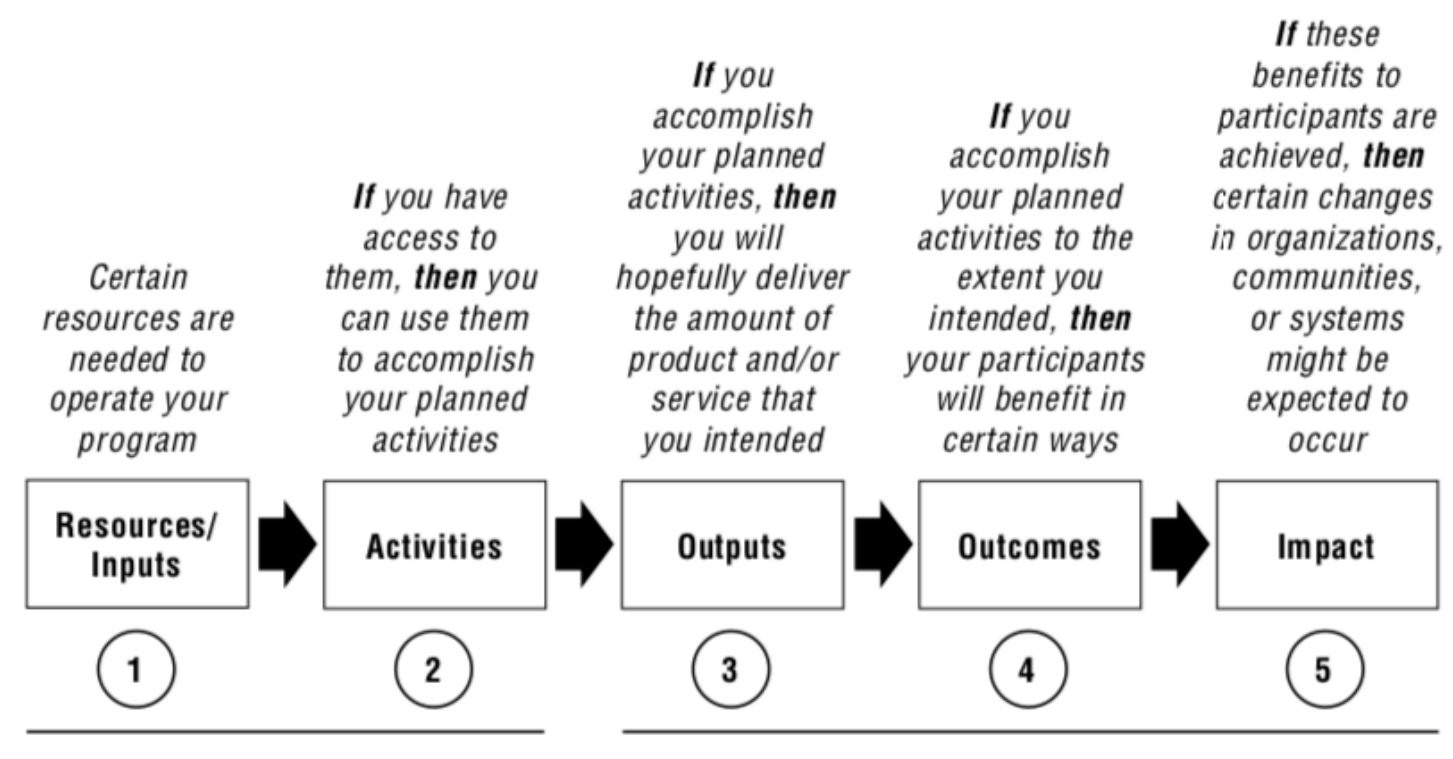

Your Planned Work Your Intended Results

Figure 2. Logic Model. (W. K. Kellogg Foundation, 2004). 
Table 1

Application of Logic Model to HNHN in a College Setting

\begin{tabular}{|c|c|c|}
\hline Resources/Inputs & Activities & Outputs \\
\hline $\begin{array}{l}\text { HNHN Grand } \\
\text { Challenges created } \\
\text { by ANA } \\
\text { - } \quad \text { Health Risk } \\
\text { Appraisal created } \\
\text { by ANA } \\
\text { - } \quad \text { RIC School of } \\
\text { Nursing is already } \\
\text { an HNHN } \\
\text { Organizational } \\
\text { Partner } \\
\text { - Undergraduate } \\
\text { - } \text { students } \\
\text { - } \quad \text { Graduate students } \\
\text { - Full time faculty }\end{array}$ & $\begin{array}{l}\text { - Presentations at } \\
\text { faculty meeting, } \\
\text { welcome reception, } \\
\text { and Student } \\
\text { Nurses' } \\
\text { Association } \\
\text { meeting } \\
\text { - Participants will be } \\
\text { asked to complete } \\
\text { Health Risk } \\
\text { Appraisal, join the } \\
\text { RIC School of } \\
\text { Nursing HNHN } \\
\text { group, and } \\
\text { participate in } \\
\text { HNHN grand } \\
\text { challenges }\end{array}$ & $\begin{array}{l}\text { Self-reported } \\
\text { participation in } \\
\text { HNHN Grand } \\
\text { Challenges, Health } \\
\text { Risk Appraisal and } \\
\text { joining the RIC } \\
\text { School of Nursing } \\
\text { HNHN group }\end{array}$ \\
\hline
\end{tabular}




\section{Method}

\section{Purpose/Research Question}

The purpose of this project was to increase participation in the ANA's HNHN Grand Challenge in a college setting with students and faculty. It addresses the question: Does promoting participation in HNHN through in-person presentations for Rhode Island College (RIC) School of Nursing undergraduate students, RN to BSN students, graduate students, and faculty increase the number of participants who join the HNHN online group for RIC and participate in a minimum of one HNHN Grand Challenge compared to pre-intervention?

HNHN and Grand Challenges were created by ANA. Rhode Island College School of Nursing was already an organizational partner, but prior to the intervention there were only five members in the group. ANA membership was not required to join HNHN or take the Health Risk Appraisal (ANA, 2018).

\section{Research Design}

The design was a program development project using the ANA's HNHN Grand Challenge adapted for implementation in a college setting.

\section{Sampling Plan and Sites}

The sample included at total of 32 sophomore, junior, and senior undergraduate

nursing students, graduate nursing students, and full-time nursing faculty at Rhode Island College School of Nursing. Participants were age 18 and older. The college has classes on two campuses and both locations were included. Only students and faculty who completed a retrospective pre/post survey were considered participants. 


\section{Procedures}

This project was reviewed by the RIC Institutional Review Board (IRB) in November 2018. The IRB determined that this project should be considered a quality improvement project rather than research and that signed informed consent was not necessary. The student researcher conducted 10 minute presentations using power point at a faculty meeting, a graduate student reception, and a Student Nurses Association (SNA) meeting in January and February 2019. Permission to present at these meetings was granted by the dean of the school of nursing (Appendix A) for the faculty meeting, the director of graduate studies (Appendix B) for the graduate student reception, and the SNA faculty advisor (Appendix C) for the SNA meeting. Presentations all used the same power point slides and included information on the purpose of HNHN, health of nurses, findings from the Healthy Nurse Survey, information on upcoming grand challenges, how to participate, and information on how other schools of nursing have implemented HNHN These included wellness committees, yoga classes, step challenges, stress relief walks, and back safety and exercise programs. Attendees were encouraged to join HNHN, take the Healthy Nurse Survey, and participate in Grand Challenges.

The dean also allowed the researcher to send one email to all students and faculty. This informational email (Appendix E) was sent by the department secretaries at the end of February and reinforced the information provided at the student and faculty meetings. The hope was that providing a link to HNHN in an email would make it easier to sign up and increase participation. The email included contact information for the student researcher. Posters (Appendix D) with information on HNHN were also placed on bulletin boards in student and faculty lounges at each campus. 
The researcher returned to a faculty meeting and a SNA meeting in April and May 2019 to provide an informational letter approved by the IRB (Appendix F) and request participation in a retrospective pre/post survey (Appendix G). Participants were informed that there were no known risks to taking the survey and that there would be no consequences for choosing not to take the survey. They were not asked any sensitive questions and were not asked any questions about results of their personal HNHN Health Risk Appraisal. The one page surveys were completed in less than five minutes.

Attendees were advised that they may choose to complete the survey or not complete the survey. Individuals who chose to participate completed a paper and pencil survey in the presence of the researchers and placed their own surveys in an envelope or handed completed surveys to one participant who volunteered to collect surveys and place them in an envelope. Surveys will be stored securely in the primary investigator's office for a minimum of three years. 


\section{Results}

According to the Logic Model, outcomes are changes in behaviors, knowledge, or skills and are measured at least one year after the after the intervention (W. K. Kellogg Foundation, 2004). This project was completed in one semester so it did not allow enough time to measure health outcomes, but it was possible to measure outputs in terms of an increase in enrollment and participation which are the immediate changes resulting from an intervention (W. K. Kellogg Foundation, 2004). Prior to the intervention, only five members were on the RIC HNHN online group so any increase was seen as a positive output. The RIC HNHN online group became two groups and it was unknown why this happened. Between the two groups, there were a total of ten members at the end of the semester.

Fifteen faculty members and 17 undergraduate students completed surveys and were included in the data analysis. The first question verified that participants had attended the presentation on HNHN at the start of the semester. Five surveys were excluded because participants indicated that they had not attended a presentation on HNHN at the start of the semester. All surveys included in the data analysis reported attending a presentation on HNHN.

Faculty members showed the greatest knowledge and participation in HNHN before the intervention with 53\% reporting previous knowledge of HNHN and 33\% reporting previously joining HNHN. One faculty member reported joining HNHN as a result of the presentation and $13 \%$ of faculty reported participating in at least one HNHN Grand Challenge after the presentation. Twenty-seven percent of faculty reported that they participated in $\mathrm{HNHN}$ because they were influenced by the presentation at the start 
of the semester. It is surprising that a greater percentage of participants reported that their participation was due to a presentation on HNHN than the percentage of participants who reported participating in HNHN. The survey did not ask for comments, but some of the surveys included comments suggesting that participants may have been inspired by HNHN to work towards their personal wellness goals rather than participating in $\mathrm{HNHN}$ Grand Challenges.

Of the 17 undergraduate participants, only $24 \%$ reported previous knowledge of HNHN and only $6 \%$ reported previously joining HNHN. They showed a greater change in participation with $6 \%$ of participants joining $\mathrm{HNHN}$ after the presentation and $12 \%$ taking the Health Risk Appraisal after the presentation. Eighteen percent of undergraduate respondents reported that they were influenced to participate in HNHN by the presentation at the start of the semester. None of the undergraduate students reported previous or current participation in any HNHN Grand Challenges.

Table 2

Results of Retrospective Pre/Post Survey

\begin{tabular}{|l|l|l|}
\hline & Faculty & $\begin{array}{l}\text { Undergraduate } \\
\text { Students }\end{array}$ \\
\hline Total number of surveys returned & 15 & 17 \\
\hline Attended HNHN presentation & $100 \%$ & $100 \%$ \\
\hline Previous knowledge of HNHN & $53 \%$ & $24 \%$ \\
\hline Previously joined HNHN & $33 \%$ & $6 \%$ \\
\hline Joined HNHN after presentation & $6 \%$ & $6 \%$ \\
\hline Previous Health Risk Appraisal & $20 \%$ & 0 \\
\hline Health Risk Appraisal after presentation & $6 \%$ & $12 \%$ \\
\hline $\begin{array}{l}\text { Previous participation in HNHN Grand } \\
\text { Challenges }\end{array}$ & $20 \%$ & 0 \\
\hline $\begin{array}{l}\text { Participated in HNHN Grand Challenges after } \\
\text { presentation }\end{array}$ & $13 \%$ & 0 \\
\hline Participated in HNHN because of presentation & $27 \%$ & $18 \%$ \\
\hline
\end{tabular}




\section{Summary and Conclusions}

Improving the health of populations by reducing and preventing noncommunicable diseases is a global and national priority. Compared to peer countries, the U.S. ranks poorly in prevalence of obesity, diabetes, heart disease, chronic lung disease, and disability (The National Academies, National Research Council and Institute of Medicine, 2013). Healthy People 2020 identified a need to improve adult American's self-reported physical and mental health (U.S. Department of Health and Human Services, 2018). According to ANA research, nurses are also struggling to achieve optimal measures of health and are more likely than the general population to be overweight, get inadequate amounts of sleep, and have high levels of stress. HNHN is ANA's program to encourage nurses to improve their health in the key areas of physical activity, rest, nutrition, quality of life, and safety (ANA, 2018).

This project aimed to determine whether promoting HNHN through in person presentations would increase participation in HNHN in a college setting. The Logic Model and Ecological Model were used as frameworks for implementing ANA's HNHN Grand Challenge. This project achieved limited success and had some limitations that should be addressed. The sample size of 32 participants was relatively small and greater participation would have been preferred, but the more significant challenges are due to environmental factors.

Results of this research should be considered in the context of the Ecological Model. Interventions focused primarily on the intrapersonal level with presentations on HNHN aiming to change the knowledge, attitudes, or behaviors of participants. All levels of the Ecological Model influence the ability of students and faculty to adopt healthier 
lifestyles and addressing more levels of the Ecological Model may have resulted in greater success of this project. Contact between the researcher and participants needs to be considered as an interpersonal factor. The intervention included limited contact and more frequent regular contact between the researcher and participants could have used social media and may have resulted in more changes in behavior. Students and faculty attended separate presentations and results were interpreted separately for students and faculty. Perhaps attending a presentation together would have allowed faculty to set an example for students and encourage students participation.

Levels of the Ecological Model that the researcher had less control over, but are important influences on health include institutional, community, and public policy factors. Institutional factors that must also be considered in the context of the Ecological Model include the university policies and infrastructure that influence any intervention implemented at the university. The university has classes on two separate campuses making it difficult to create an atmosphere where interpersonal processes encourage participation in HNHN. The off campus Nursing Education Center is managed by an outside building management company so there are restrictions on where posters can be displayed in the building. The building has television (TV) screens in hallways that display rotating announcements and information. A request to post information on HNHN on these TV screens was denied. This project relied on the university email for communication and emails to students and faculty were sent from secretaries rather than being sent directly from the researcher. Another limitation was difficulty communicating with students and faculty to get the message out about HNHN. Communicating with the SNA leadership to schedule presentations at meetings was difficult. The researchers 
communicated with students and faculty only through email and perhaps another form of communication may have been more effective.

Since the research proposal was submitted to the IRB, that also became an institutional factor in this project. The project had to include only what was submitted to the IRB. More opportunities became available during the course of the project including a webinar related to HNHN and the researcher would have liked to offer an opportunity for students and faculty to view this as a group. There was also an opportunity to collect surveys from graduate students at poster presentations in May, but this had not been included in the proposal submitted to the IRB.

The relationships between RIC School of Nursing and ANA and HNHN are community factors relevant to this project. Although there was an increase in membership in the RIC School of Nursing HNHN online group, the relationships remained passive with RIC School of Nursing members only receiving information from HNHN. Getting this information to people who are not requesting it remains a challenge for HNHN and institutions including RIC School of Nursing.

The HNHN presentation at the faculty meeting sparked more discussion than either of the other presentations. There was a question at the faculty meeting about a 2018 RIC school of nursing honors project on the health of student nurses. The faculty member who advised that project emailed the paper to all faculty after the meeting. There was interest in integrating this into curriculum, but results did not suggest that this happened. 


\section{Recommendations and Implications for Advanced Nursing Practice}

This project resulted in minimal changes in behavior of students and faculty, but has important implications for advanced practice nursing related to education, practice policy, and research. Although this project did not assess nurses' or student nurses' knowledge about health promotion, previous research has identified a great need for improved knowledge of health promotion among nurses and student nurses, and some important topics could be incorporated into nursing curriculums. Both Blake et al., (2011) and Blake and Harrison (2013) found that nursing students need more education on recommendations for physical activity, nutrition, and safe use of alcohol (Blake et al., 2011; Blake \& Harrison, 2013). Rock's (2018) research should also be incorporated into nursing curriculums with education on stress management and the importance of taking preventative action to prevent disease (Rock, 2018). Even with a greater emphasis on health promotion in nursing curriculums, the health behaviors of students and faculty may not change so it is essential for advanced practice nurses to address the environments where nurses and students make choices about health promotion.

Advanced practice nurses need to engage nurses at all levels of practice including student nurses and administrators to improve their own health behaviors and the health of the nursing workforce. Individual barriers to $\mathrm{HNHN}$ participation were not addressed in this project, but nurses need to advocate for institutional policies that allow for time and flexibility to participate in HNHN and maintain healthy behaviors. It is essential for institutions to create an environment of health rather than isolated health promotion interventions (Kent et al., 2016). Employers need to take responsibility for the health of nurses (Kelly et al., 2016), and advanced practice nurses can make administrators more 
aware of the need for this by sharing data from ANA's Health Risk Appraisal. Advanced practice nurses need to advocate for environmental changes to workplaces that make healthy choices easy choices by making healthy foods available and creating comfortable break areas (Nicholls et al., 2016). Fresh food is available in a cafeteria on the main RIC campus and a snack bar at the off campus nursing education building so healthy options are available. Both locations also have vending machines and removing sugar sweetened beverages and candy could help students and faculty make healthier choices.

This project relied on educational presentations to encourage individual participation in HNHN Grand Challenges. Other schools of nursing featured on the HNHN blog have offered opportunities to participate in Grand Challenges as a group (ANA, 2018). It could be helpful for advanced practice nurses to offer a group activities in college or workplace settings as a way to get people started with HNHN and then assess individual participation in grand challenges after the group activity.

This project lacked changes to the environment that would make healthy choices easy choices. According to Frieden's (2010) Health Impact Pyramid, counseling and education make up the top tier of the five tier pyramid. This level of the pyramid requires increased individual effort, but has the smallest population impact. When the Health Impact Pyramid is applied to this project, it is not a surprise that reported changes in behavior were minimal. The bottom tier of the pyramid is interventions that address socioeconomic factors. These interventions have the smallest individual effort and the greatest population impact. Following this tier are interventions that change the environment to make health decisions the default (Frieden, 2010). A more successful application of HNHN in a college or workplace setting needs to better incorporate the 
Ecological Model and include changes to the environment that would make healthier nutrition and increased physical activity the default choices (McLeroy et al., 1988).

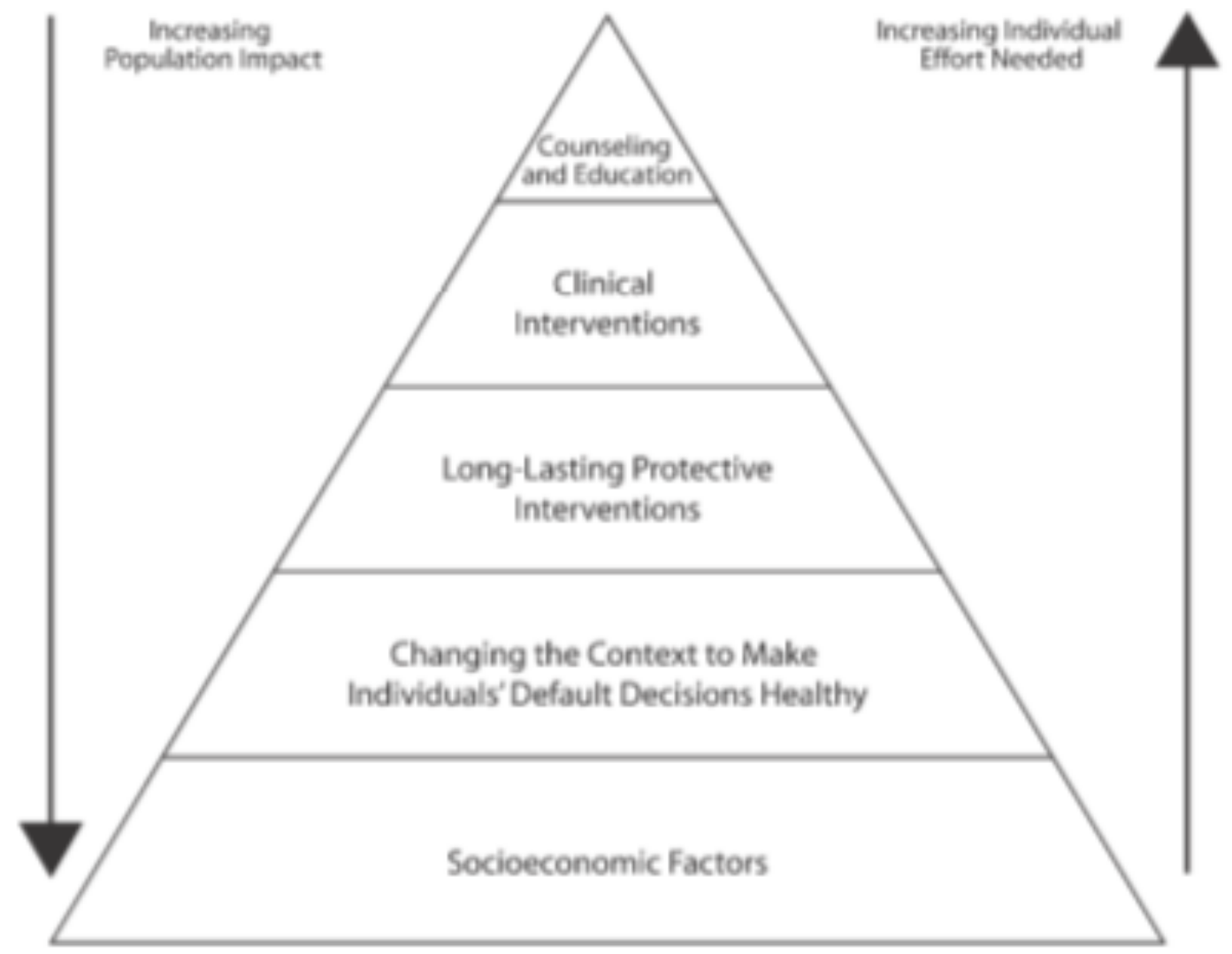

Figure 3. Health Impact Pyramid. (Frieden, 2010).

Transportation should be addressed as a public policy and institutional policy. Several of the universities in this city encourage use of public transportation by providing free bus passes for students and faculty. While this project was conducted, this university only offered half price bus passes for students and had free parking passes available for both campuses. The semester after HNHN was implemented at RIC School of Nursing, this university also started offering free bus passes for students. Providing this financial incentives to encourage use of public transportation could have a greater impact on increasing activity than educational presentations on physical activity. 
Advanced practice nurses should be leaders in improving the health of nurses by conducting research and quality improvement projects. Results of this project showed some small positive changes for both students and faculty suggesting that continuing to promote HNHN with some modifications may be helpful in college settings. Faculty reported greater knowledge and participation in HNHN prior to the intervention, but students reported greater changes in participation after the intervention. Since the Logic Model states that outcomes should be measured at least one year after the intervention (W. K. Kellogg Foundation, 2004), it would be worthwhile to replicate this project and continue it for at least one year. Research is needed to determine whether there is a correlation between increasing nurses' and student nurses' knowledge about health promotion and their personal health behaviors. ANA believes that healthier nurses have the potential to improve the health of the population by modeling healthier behavior and being more credible as coaches (ANA, 2018). However, more research is needed on the impact of nurses as role models (Kelly et al., 2016) and the impact of nurses' personal health behaviors on their health promotion practices with patients (Kelly et al., 2017).

In order to really make a population impact, HNHN needs to find ways to inspire nurses to advocate for policies that create environments where the healthy choice is the default choice. Nurses are taught to advocate for the needs of individual patients, but we need to teach nurses to advocate for the nursing profession and for the health of the general population. 


\section{References}

American College Health Association. (2018). Ecological Model. Retrieved from https://www.acha.org/HealthyCampus/Implement/Ecological_Model/HealthyCam pus/Ecological_Model.aspx?hkey=f5defc87-662e-4373-8402-baf78d569c78

American Nurses Association (2015). Code of ethics for nurses with interpretive statements. Retrieved from https://www.nursingworld.org/coe-view-only American Nurses Association (2018). About the HNHN GC.

Retrieved from http://www.healthynursehealthynation.org/en/about/about-the$\underline{\text { hnhn-gc/ }}$

Blake, H. \& Harrison, C. (2013). Health behaviours and attitudes towards being role models. British Journal of Nursing, 22(2), 86-94.

Blake, H., Malik, S., Mo, P. K. H., \& Pisano, C. (2011). "Do as I say, but not as I do": Are next generation nurses role models for health? Perspectives in Public Health, 131(5), 231-239. doi: 10.1177/1757913911402547

Bronfenbrenner, U. (1977). Toward an experimental ecology of human development. American Psychologist, 513-531.

Cane, J., O’Connor, D., \& Michie, S. (2012). Validation of the theoretical domains framework for use in behavior change and implementation research. Implementation Science, 7(37). doi: 10.1186/1748-5908-7-37

Darch, J., Baillie, L., \& Gillison, F. (2017). Nurses as role models in health promotion: A concept analysis. British Journal of Nursing, 26(17), 982-988.

Frieden, T. R. (2010). A framework for public health action: The health impact pyramid. 
American Journal of Public Health, 100(4), 590-594. doi:

10.2105/AJPH.2009.185652

Fronteira, I. \& Ferrinho, P. (2011). Do nurses have a different physical health profile? A systematic review of experimental and observational studies on nurses' physical health. Journal of Clinical Nursing, 20, 2404-2424. doi: 10.1111/j.13652702.2011.03721.x

Goetzel, R. Z., Mosher Henke, R., Tabrizi, M., Pelletier, K. R., Loeppke, R., Ballard, D. W., . . \& Metz, R. D. (2014). Do workplace health promotion (wellness) programs work? Journal of Occupational and Environmental Medicine, 56(9), 927-934. doi:10.1097/JOM.0000000000000276

Kelly, M., Wills, J., Jester, R., \& Speller, V. (2016). Should nurses be role models for healthy lifestyles? Results from a modified Delphi study. Journal of Advanced Nursing, 73(3), 665-678. doi: 10.1111/jan.13173

Kelly, M., Wills, J., \& Sykes, S. (2017). Do nurses' personal health behaviours impact on their health promotion practice? A systematic review. International Journal of Nursing Studies, 76, 62-77. doi: 10.1016/j.jjnurstu.2017.08.008

Kent, K., Goetzel, R., Roemer, E., Prasad, A., \& Freundlich, N. (2016). Promoting health workplaces by building cultures of health and applying strategic communications. Journal of Occupational and Environmental Medicine, 58(2), 114-122. doi: 10.1097/JOM.0000000000000629

Malik, S., Blake, H., \& Suggs, S., (2014). A systematic review of workplace health promotion interventions for increasing physical activity. British Journal of Health Psychology, 19,149-180. doi: 10.1111/bjhp.12052 
Maselli, M., Ward, P., Gobbi, E., \& Carraro, A. (2018). Promoting physical activity among university students: A systematic review of controlled trials. American Journal of Health Promotion, 20(10), 1-11. doi:10.1177/0890117753798

McLeroy, K., Bineau, D., Steckler, A., \& Glanz, K. (1988). An ecological perspective on health promotion programs. Health Education Quarterly, 15(4), 351-377.

Michie, S., Johnston, M., Abraham, C., Lawton, R., Parker, D., \& Walker. A. (2005). Making psychological theory useful for implementing evidence based practice: A consensus approach. Quality and Safety in Helath Care, 14. 26-33. doi:

10.1136/qshc. 2004.011155

Nicholls, R., Perry, L., Duffield, C., Gallagher, R., \& Pierce, H. (2016). Barriers and facilitators to healthy eating for nurses in the workplace: an integrative review. Journal of Advanced Nursing, 73(5),1051-1065. doi: 10.1111/jan.13185

Power, B. T., Kiezebrink, K., Allan, J. L., \& Campbell, M. K. (2017). Understanding perceived determinants of nurses' eating and physical activity behaviour: A theory-informed qualitative interview study. BMC Obesity, 4(18), doi: $10.1186 / \mathrm{s} 40608-017-0154-4$

Rock, L. (2018). Assessment of the level of health promotion among undergraduate nursing students at Rhode Island College: A quantitative and qualitative analysis (Unpublished honors thesis). Rhode Island College, Providence, RI.

The National Academies, National Research Council and Institute of Medicine. (2013). U.S. health in international perspective: Shorter lives, poorer health. Retrieved from https://www.nap.edu/catalog/13497/us-health-in-international-perspectiveshorter-lives-poorer-health 
U.S. Department of Health and Human Services, Centers for Disease Control and Prevention, National Center for Health Statistics. (2011). Healthy eating index2005 total and component scores for adults aged 20 and over: national health and nutrition examination survey, 2003-2004. Retrieved from https://www.cdc.gov/nchs/data/nhsr/nhsr044.pdf

U.S. Department of Health and Human Services, Centers for Disease Control and Prevention, National Center for Health Statistics. (2018). State variation in meeting the 2008 federal guidelines for both aerobic and muscle-strengthening activities through leisure-time physical activity among adults aged 18-64: United States, 2010-2015. Retrieved from https://www.cdc.gov/nchs/data/nhsr/nhsr112.pdf

U.S. Department of Health and Human Services. (2018). Healthy People 2020. Retrieved From https://www.healthypeople.gov/sites/default/files/HP2020Framework.pdf

White, M. I., Dionne, C. E., Warje, O., Koehoorn, M., Wagner, S. L., Schultz, I. Z., . . \& Wright, M. D. (2016). Physical activity and exercise interventions in the workplace impacting work outcomes: A stakeholder-centered best evidence synthesis of systematic reviews. International Journal of Occupational and Environmental Medicine, 7, 61-74.

W. K. Kellogg Foundation. (2004). Logic model development guide. Retrieved from:https://www.bttop.org/sites/default/files/public/W.K.\%20Kellogg\%20Logic $\underline{\text { Model.pdf }}$ 
World Health Organization. (2014). Basic Documents, Forty-eighth edition. Retrieved from: http://apps.who.int/gb/bd/PDF/bd48/basic-documents-48th-editionen.pdf\#page $=7$ 


\section{Appendix A}

\section{RHODE ISLAND COLLEGE}

October 8, 2018

RIC IRB Committee

600 Mount Pleasant Ave

Providence, RI 02908

Dear Committee Members

I am writing to verify my support of Emily George's MSN project "Increasing Participation in the American Nurses Association's Healthy Nurse Healthy Nation Grand Challenge in a College Setting". Emily has my permission to contact students and faculty, present at a faculty meeting and a Student Nurses Association meeting, post flyers and survey students and faculty.

The SON values student participation in research. I fully support Emily's project. Please feel free to contact me with any questions regarding this request.

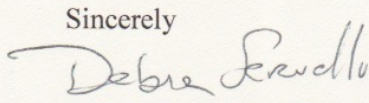

Debra Servello, DNP, APRN

Interim Dean 


\section{Appendix B}

$\frac{\text { RHODE ISLAND COLLEGE }}{\text { SCHOOL OF NURSING }}$

October 11, 2018

Dear Rhode Island College IRB:

I am writing this letter to indicate my support for Rhode Island College graduate student Emily George's master's project, Increasing Participation in the American Nurses Association's Healthy Nurses Healthy Nation Grand Challenge in a College Setting. The School of Nursing is grateful for Emily providing graduate students with a brief presentation of the ANA Healthy Nurses Healthy Nation at the January 2019 Graduate Welcome Back Event. We will do all we can to facilitate this project and believe it will benefit our students. Emily has our full support in this endeavor. Please contact me at jcostello@ric.edu if any other information is needed to further this project.

Sincerely,

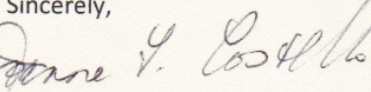

Joanne F. Costello, MPH, PhD, PHCNS-BC

Professor and Interim Graduate Chair

RI College School of Nursing

600 Mt. Pleasant Avenue • Providence, RI 02908-1996 • Undergraduate (401) 456-8013 • TTY/TDD: 711 


\section{Appendix C}

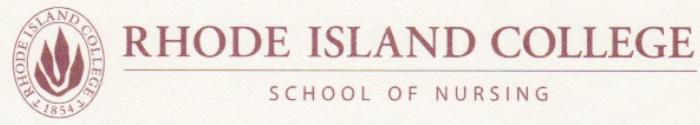

To Whom It May Concern:

November 1, 2019

I am writing in support of the project presented to me by Emily George. She would like to attend a Student Nurse Association meeting in January or February 2019 to make a brief presentation about Healthy Nurse Healthy Nation. She will then return to an additional SNA meeting in April 2019 to do a survey.

Sincerely,

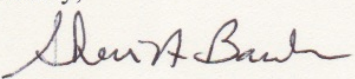

Sheri A. Boucher, MSN, RN

Assistant Professor, Nursing

Rhode Island College, School of Nursing

Fogarty Life Science, Room 112

600 Mount Pleasant Avenue

Providence, RI 02908 


\section{Appendix D}

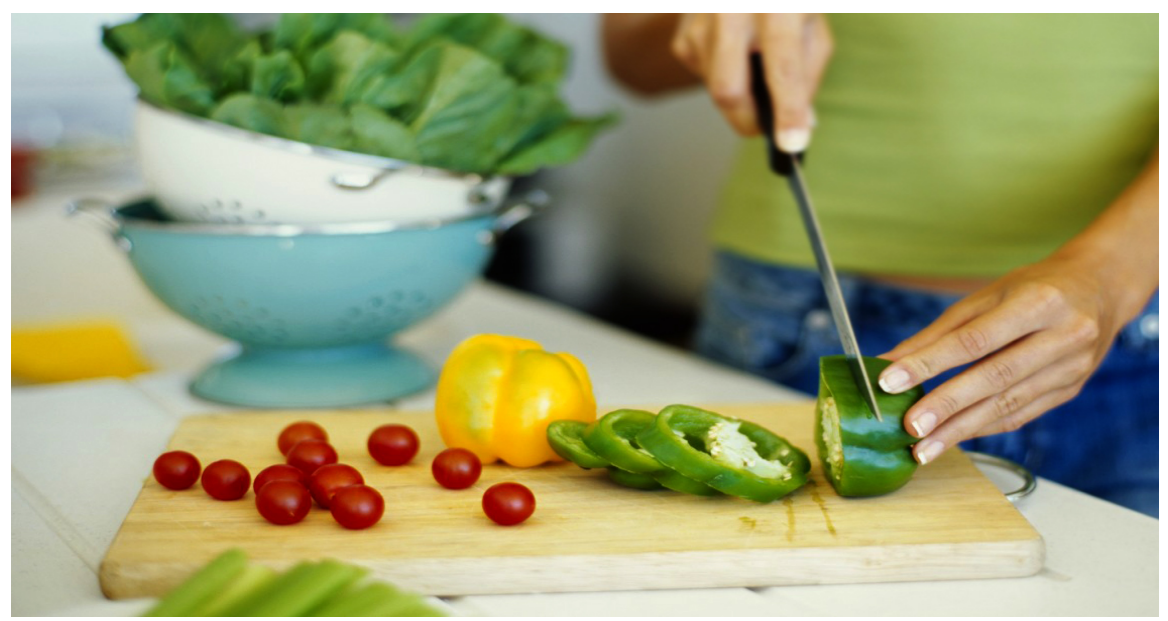

\section{Healthy Nurse Healthy Nation at Rhode Island College}

\section{http://www.healthynursehealthynation.org}

Healthy Nurse Healthy Nation is the American Nurses Association's (ANA) strategy to encourage nurses to improve their health in the key areas of physical activity, nutrition, rest, quality of life, and safety. According to the ANA Health Risk Appraisal the average BMI of nurses who responded was 28.8 and only $14 \%$ of respondents reported eating at least five servings of fruit and vegetables per day. A healthier nursing workforce has the potential to improve the health of the general population by modeling healthy behaviors for patients and the public.

\section{How to get involved:}

- Join Healthy Nurse Healthy Nation

- Take the Healthy Nurse Survey

- Participate in Grand Challenges

- Encourage your colleagues to participate

Contact: Emily George at egeorge_6976@email.ric.edu 


\section{Appendix E}

Hello SON students and faculty:

RIC SON is participating in Healthy Nurse Healthy Nation this semester. Healthy Nurse Healthy Nation is the American Nurses Association's (ANA) strategy to encourage nurses to improve their health in the key areas of physical activity, nutrition, rest, quality of life, and safety. According to the ANA Healthy Nurse Survey, the average BMI of nurses who responded was 28.8 and only $14 \%$ of respondents reported eating at least five servings of fruit and vegetables per day. A healthier nursing workforce has the potential to improve the health of the general population by modeling healthy behaviors for patients and the public. We hope that you will check out the Healthy Nurse Healthy Nation website and participate in some of the upcoming Grand Challenges.

Please visit this link to learn more about Healthy Nurse Healthy Nation: http://www.healthynursehealthynation.org

How to get involved:

- Join Healthy Nurse Healthy Nation

- Take the Healthy Nurse Survey

- Participate in Grand Challenges

- Encourage your colleagues to participate

Upcoming Grand Challenges:

- February: Tips for Healthy Sleep

- March: Curb Your Sweet Tooth

- April: Resiliency

Below is a link to a 2018 RIC SON honors project on health promotion among nursing students at RIC. Several people have requested this.

https://digitalcommons.ric.edu/honors_projects/147/

Sincerely,

Emily George and Lynn Blanchette

Contact: Emily George at egeorge_6976@email.ric.edu 


\section{Appendix F}

\section{INFORMATIONAL LETTER Rhode Island College}

Increasing Participation in the American Nurses Association's Healthy Nurse Healthy Nation Grand Challenge in a College Setting

You are being asked to be in a quality improvement project about increasing participation in the American Nurses Association's Healthy Nurse Healthy Nation (HNHN) Grand Challenge in a college setting. Participation in this project is voluntary and it is anticipated that you would be involved for up to five minutes. You are being asked because you are a student or faculty member at Rhode Island College School of Nursing. Please read this form and ask any questions that you have before choosing whether to be in the project.

Emily George, a graduate student in nursing, is conducting this quality improvement project in collaboration with the faculty advisor Lynn Blanchette, a professor at Rhode Island College.

\section{Why this Project is Being Done (Purpose(s)}

We are asking you to participate in this survey to assess whether promoting participation in HNHN through in-person presentations for Rhode Island College School of Nursing undergraduate students, RN to BSN students, graduate students, and faculty increase the number of participants who join the RIC HNHN online group and participate in a minimum of one HNHN Grand Challenge compared to pre-intervention.

\section{What You Will Have to Do (Procedures)}

If you choose to be in the project, we will ask you to:

- Confirm that you attended a Healthy Nurse Healthy Nation presentation at the beginning of the semester. If you did not attend, you are not eligible to participate in this survey.

- You will read and answer some survey questions. The questions ask about your past participation in Healthy Nurse Healthy Nation Grand Challenges and the Health Risk Appraisal. You may choose not to answer any questions you are not comfortable answering. This will take up to five minutes of your time.

\section{Risks or Discomforts}

The risks of participating in this project are minimal, meaning that they are about the same as what you would experience during your typical daily activities.

\section{Deciding Whether to Be in the Project}

Being in the project is your choice to make. Nobody can force you to do this project. You can choose not to be in the project, and nobody will hold it against you. You can change your mind and quit the study at any time, and you do not have to give a reason. If you decide to quit later, nobody will hold it against you.

How Your Information will be Protected

Data from this survey will be anonymous. 
Who to Contact

You can ask any questions you have now. If you have any questions later, you can contact Emily George at egeorge_6976@email.ric.edu or Lynn Blanchette at lblanchette@ric.edu.

You will be given a copy of this form to keep. 
Appendix G

INCREASING PARTICIPATION IN THE AMERICAN NURSES ASSOCIATION'S HEALTHY NURSE HEALTHY NATION GRAND CHALLENGE IN A COLLEGE SETTING

Please choose one: undergraduate student RN to BSN student graduate student faculty 1. Did you attend the presentation about Healthy Nurse Healthy Nation given at the start of this semester? (If not, thank you. You have completed the survey.)

Yes $\quad$ No

2. Did you know anything about HNHN before the HNHN presentation given at the start of this semester?

Yes $\quad$ No

3. Had you signed up for HNHN before the presentation given at the start of this semester?

Yes $\quad$ No

4. If not, did you sign up for HNHN as a result of the presentation given at the start of this semester?

Yes $\quad$ No

5. Had you taken the Health Risk Appraisal before the presentation given at the start of this semester?

Yes No

6. If not, did you take the Health Risk Appraisal as a result of the presentation given at the start of this semester?

Yes No

7. Did you participate in any HNHN Grand Challenges before the presentation given at the start of this semester?

Yes $\quad$ No

8. Did you participate in any HNHN Grand Challenges as a result of the presentation given at the start of this semester?

Yes No

9. If you did participate in HNHN, was your decision influenced by the presentation given at the start of the semester?

Yes No 
